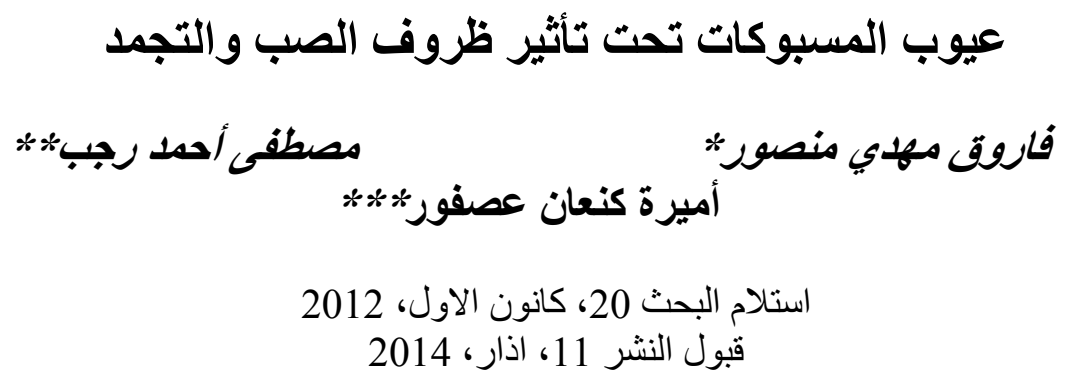

الخلاصهة:

أوضحت نتائج البحث الى التوصل التهل الى الظروف التي تمنع من ظهور الفجوات الابتدائية أو الثانوية وتحقيق

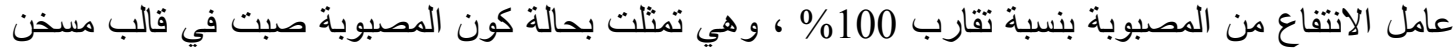

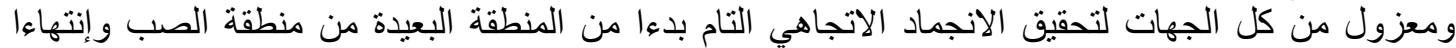

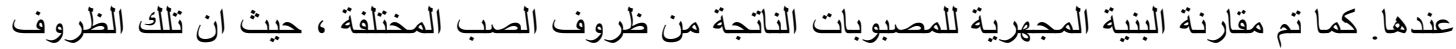

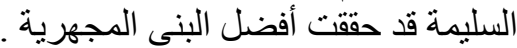

الكلمات مفتاحية: المسبوكات، ظروف الصب والتجمد، الانجماد الاتجاهي.

\begin{abstract}
= P و

ولغرض تحديد مو اصفات عناصر منظومة الصب كنب

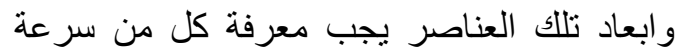

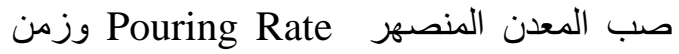

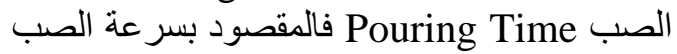
هي القوة التي تؤدي الى تدفق المنصهر من البودة التهة الىى تجويف القالب وهي نالتي التئة بفعل التعجيل

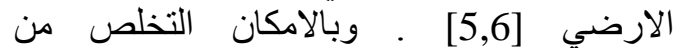
الاضطر اب للمعدن المنصهر عند سكبه في تجويف القالب من خلال استخدام راس مصب الإن السبك بحيث فيث يصغر قطره من الاسفل وفي بعض التض الاحيان تستخدم اكثر من قناة في حالة المصبو الاسبات الكبيرة .

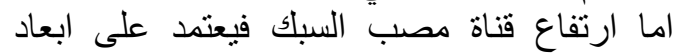

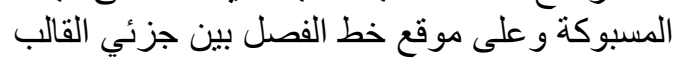
فعندما يكون نظام الصب غيل غير ضغطي فال فان مساحة

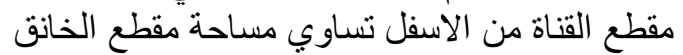
Choke فان مساحة مقطع القناة تكون اكبر من مجمون

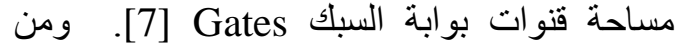
الجدير بالملاحظة ان الثكل المنحني لقناة مجرى التئ السبك Runner يسبب الاضطراب في مجرى الإن الإن

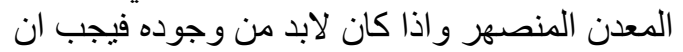
تكون مساحة المقطع له اكبر مما هو لهان عليه للثنكل

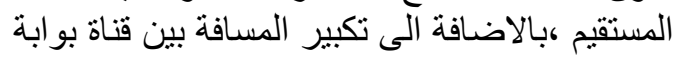
السبك وطرف القناة المحنية.

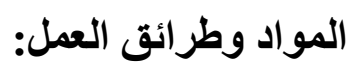

تناول البحث ثلاث محاور أساسية:

1-ظروف الصب والتجمد لسبيكة الديور الومن،

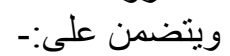

* الصب في قالب غير مسخن و غير معزول . .
ان الغاية الاساسية من تصميم المصبوبات هو الموبة المبات

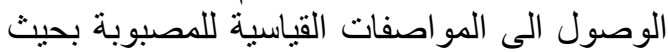

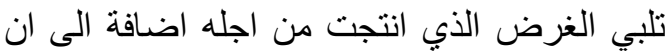

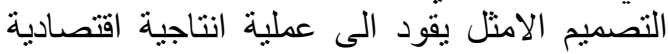

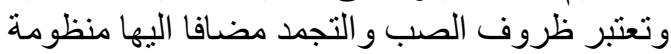

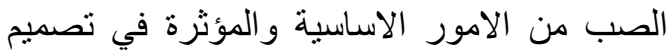

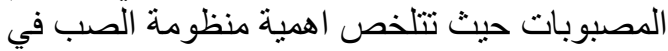

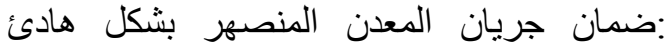

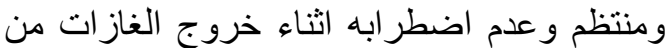
تجويف القالب مما يؤدي الى عدم تعرية السطح

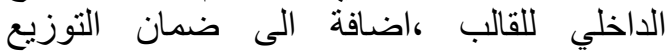
الحراري المتجانس لحين ملئ تجاويف القالب التئي

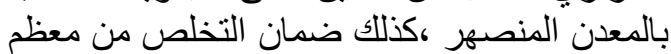

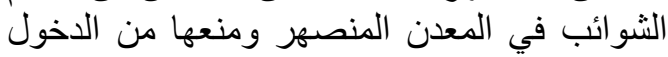

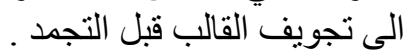
ومن الجدير بالذكر ان منظومة الصب الصب الجيدة

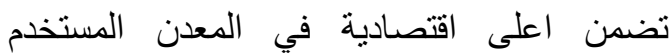

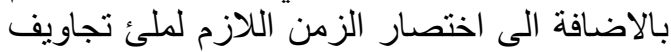

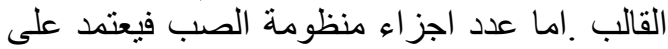

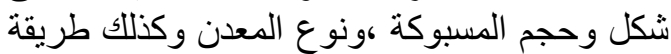
السباكة ،ففي بعض المبركة المسبوكات الكبيرة الحجم

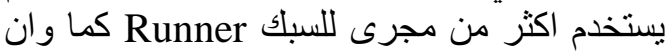

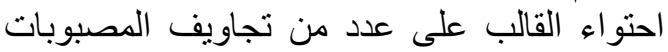

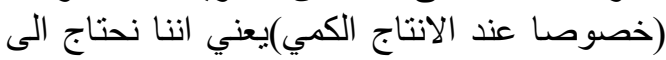

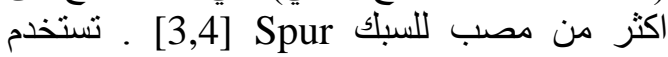
معادلة رينولا Reynolds لتحديد نوعية التونية الجريان لمكيصهر المعدن المراد ملئ تجاويف القالب به نبه

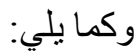
$\mathrm{R}_{\mathrm{e}}=\mathrm{V} . \mathrm{d} .(\mathrm{p} / \mathrm{v})$

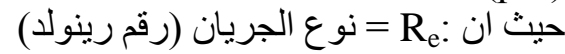
معدل السرعة =V

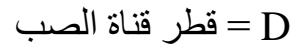


تتم عملية الصهر يتم وضع القالب الساخن وتثبيته

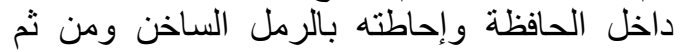
وضع الغطاء المجمع مع المزدوجات بات الحرلة الحرارية و إعادة عملية الصب على نفس الخطو الئ السابقة .

جلول (1) يبين التركيب الكمياوي للالمنيوم النقي التجاري.

\begin{tabular}{|c|c|c|c|c|c|c|}
\hline $\mathbf{S i}$ & $\mathrm{Fe}$ & Mn & Zn & $\mathbf{C u}$ & Al & العنصر \\
\hline 0.043 & 0.16 & 0.09 & 0.017 & 0.09 & 99.6 & النسبة\% \\
\hline
\end{tabular}

\section{النتائج و المناقثة :}

اجريت الدراسة على سبيكة الديور المين وباربعة

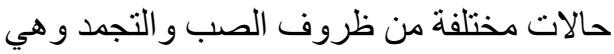

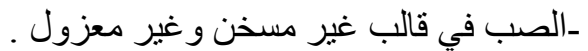

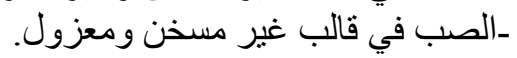
ـ الصب في قالب مسخن غير معزول . ـ الصب في قالب مسخن معزول. فالتسخين للقالب كان بدرجة (400)خُ ،الما العزل

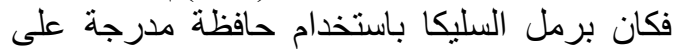

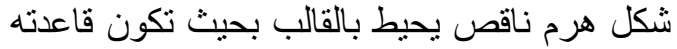

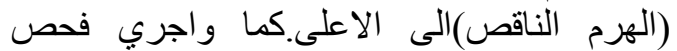
للبنية المجهرية للمصبوبات بلىميع الإلى الحالات السابقة الذكر للتعرف على مدى تاثير البنية المجهرية التهاتية بظروف الصب والتجمد

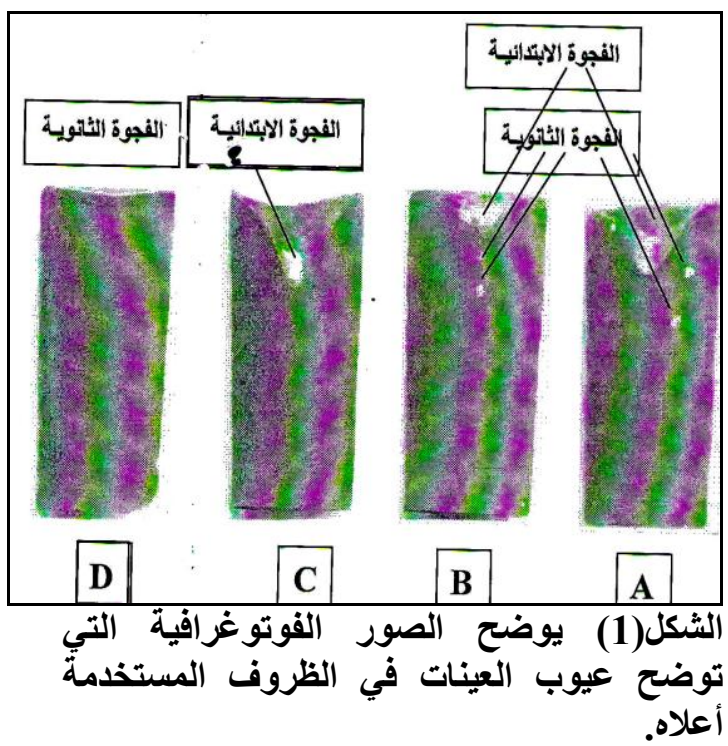

معزول. تزداد درجة حرارة المصبوبة باتجاه

الأعلى و السبب يعود الى سحب جداد درار القالب السفلي بائي

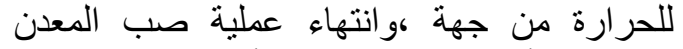
السائل من أعلى القالب من جهة أخرى ،وبعدة من مرور

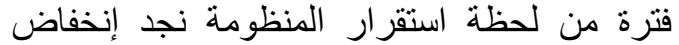
درجة الحرارة عند قمة المصبوبة ومن ثم حصول المنظ
* الصب في قالب غير مسخن ومعزول. * الصب في قالب مسخن غير معزول فيز معزول. * الصب في قالب مسخن معزول. 2-البنية المجهرية لسبيكة الديور الومن بإختلاف الظروف السابقة. 3-عيوب المصبوبات لسبيكة الديور الومن بإختلاف

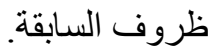

إستخدام فرن المقاومة الكهربائية الذي تصل

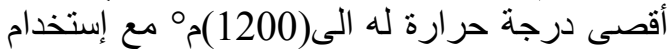
بوتقة كرافيتية في عملية الصهر. ونم الصارة إستخدام الالمنيوم ذي النقاوة التجارية (99.6)\% تحليله الكمياوي في الجدول (1)، و الذي تم تحليله

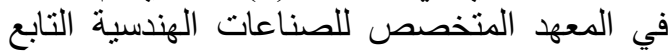
لوزارة الصناعة والمعادن. وقد كان على شلى شكل الكل

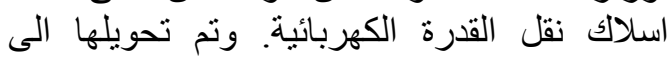

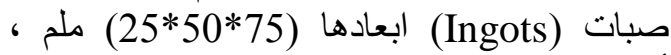
أستخدمت هذه الصبات لعمل سبيكة الاساس بعاس بعد

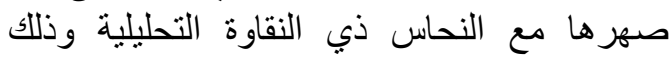
بصهر الالمنيوم ورفع درجة حرارته الى (900)

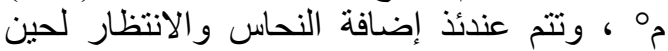
ذوبانه في السبيكة ومن ثم صب إنب السبيكة الاساس على شكل مصبوبات بنفس الابعاد السابقة. بعد ذلك اللك

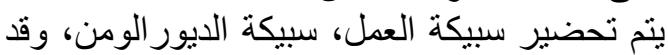
تم التحضير هذا بخلط أوزان محسوبة من سبيكة الإيكة

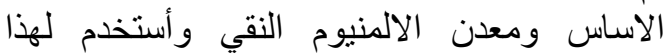
الغرض ميزان حساس ، وعند الإلثير تبريد هذه السبيكة

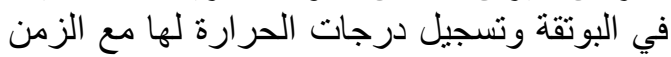

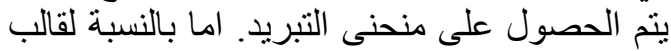

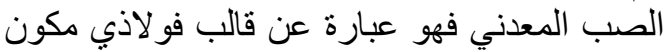

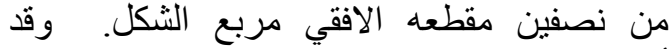

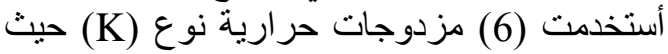

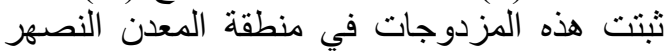

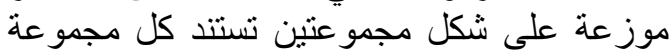

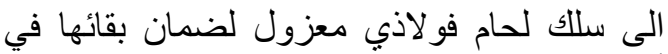

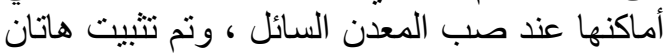

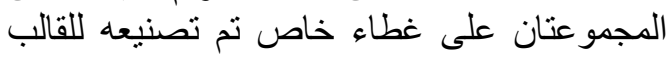

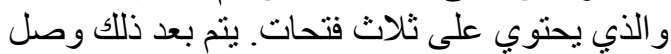

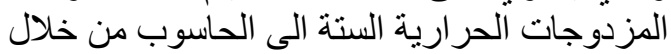
بطاقة خاصة (AD-Converter). وبعدها لئها يتم

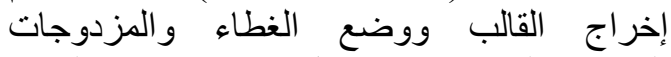
الحرارية ليتم بعدها تسجيل درجة حرارة النطاة الجدار

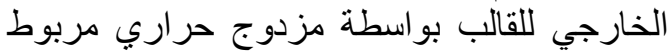

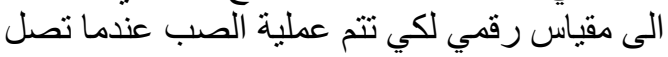

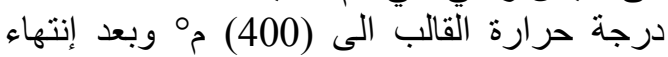

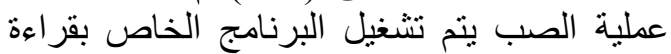
وتسجيل درجات الحرارة. اما بالنسبة للعينة الثانية الثانية

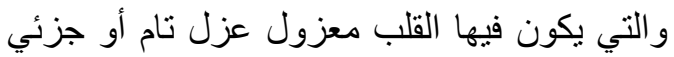

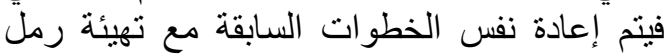
السليكا وتسخينه الى درجة (300) مثْ ــ وبعد ان 


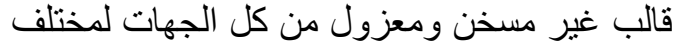

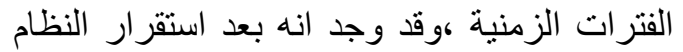

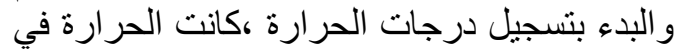

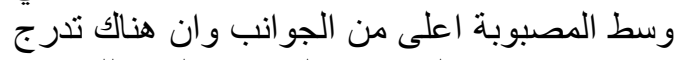
حراري باتجاه المركز واعلى الثبوت على التى ذلك عند عند

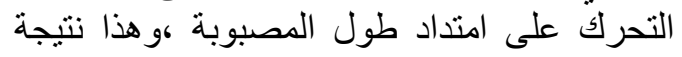

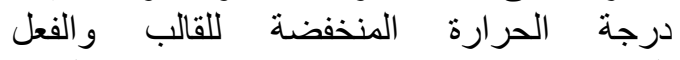
التصقيعي Chilling Effect لان فقدان الطاقة يكون من خلال ملامسة جدران القالب للهواء للاء الطاء الجوي بطبقة التوصيل. 3- الصب في قالب مسخن و غير معزول:

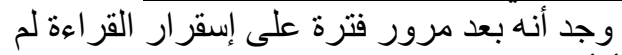

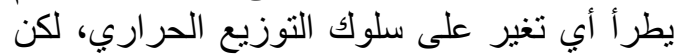

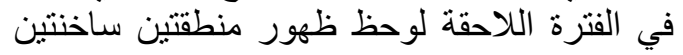

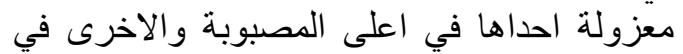

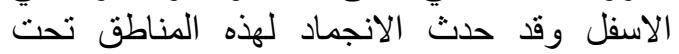
ظروف لايمكن التعويض عن المعدن المنكمش فيها

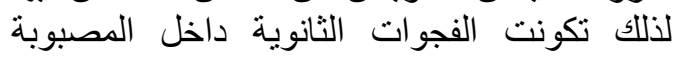

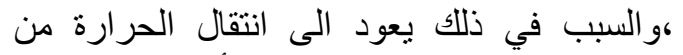
الجدران الجانبية للقالب الذي أدى النى الى تركز

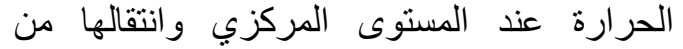

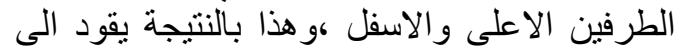
تحقيق اتجاهين مفضلين للانتقال الحرارة الإة ولو بشكل

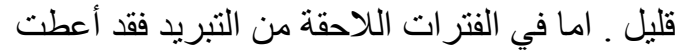

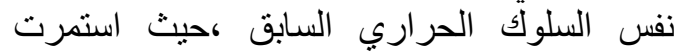

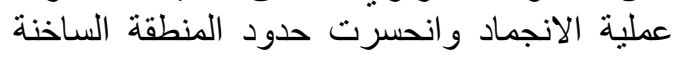
في مركز المصبوبة في الموقعين العلوي والسفي السفلي

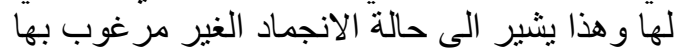

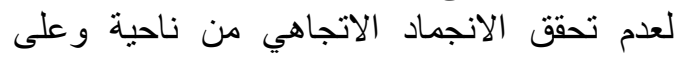
امكانية تغذية المناطق المتجمدة في النهاية من ناحية

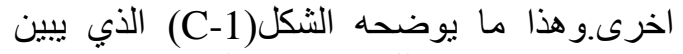

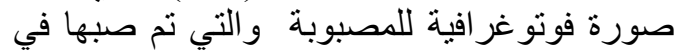

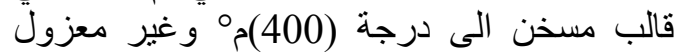

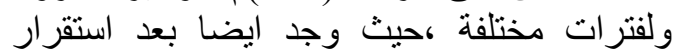

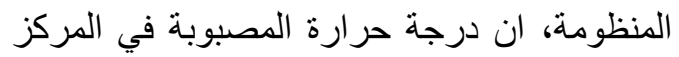

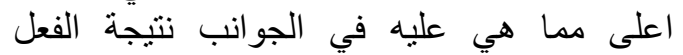
التصقيعي للقالب واثره في فحب في الجي وتبديد الحرارة

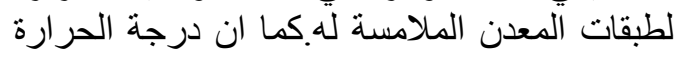

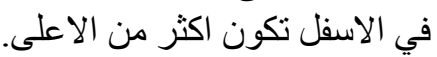

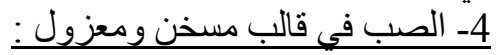

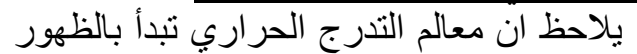

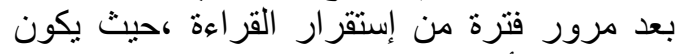

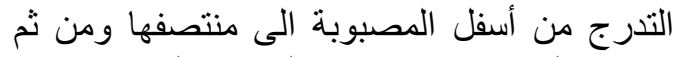

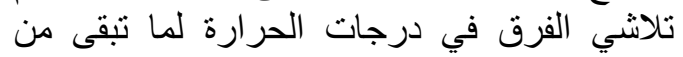

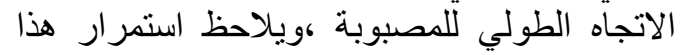

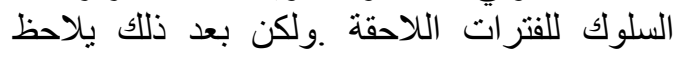

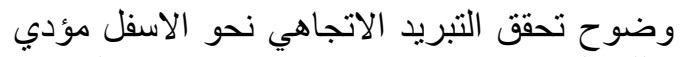

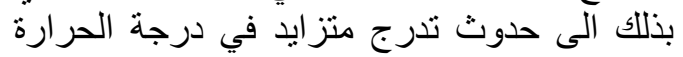

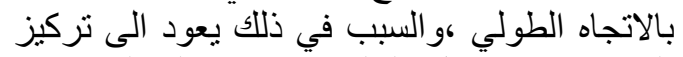
الحرارة من أسفل القالب بالاتجاه الطولي يكون
تدرج منزايد باتجاه الجدار السفلي للقالب ،ونتيجة

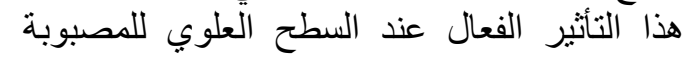

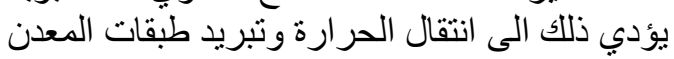

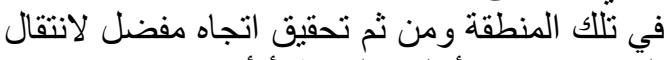

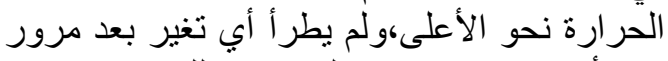

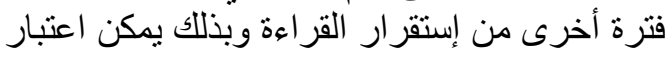

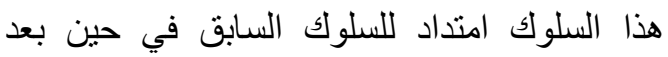

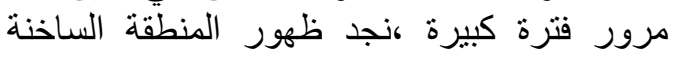

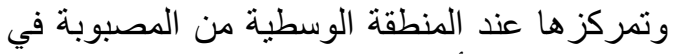

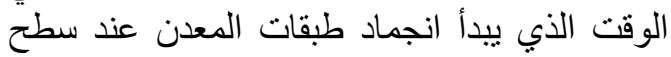

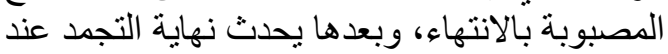

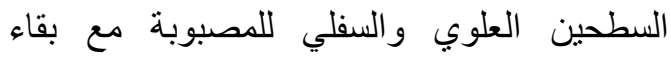

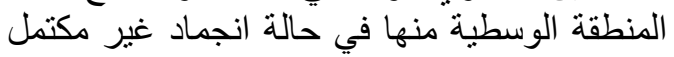

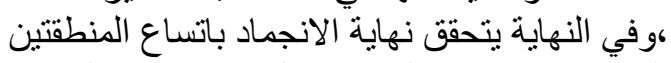

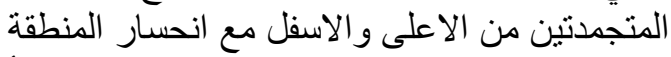

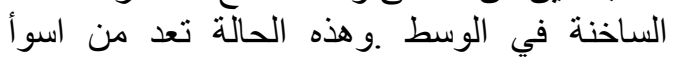

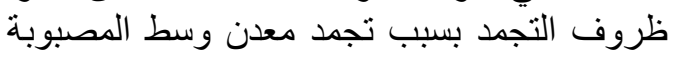

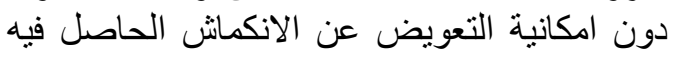

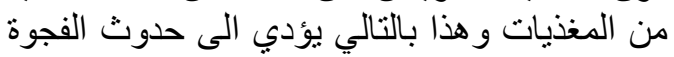

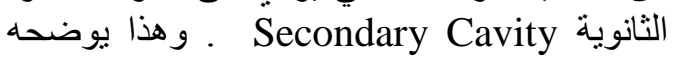
الثكل(A) الثيانوبة الذي يبين الصورة الفوتوغرافية

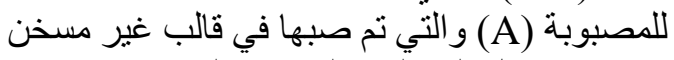

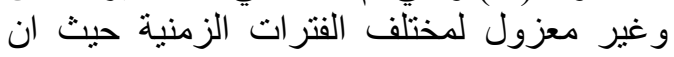

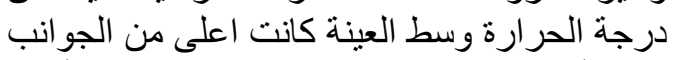

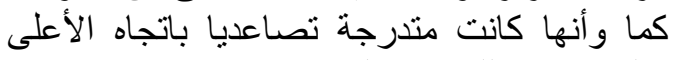

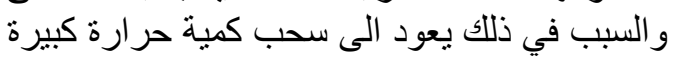

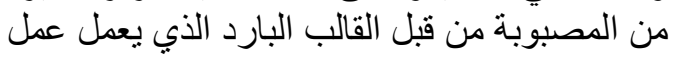

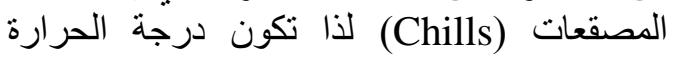

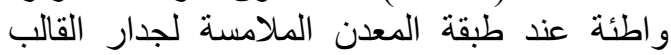

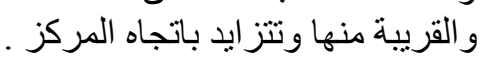

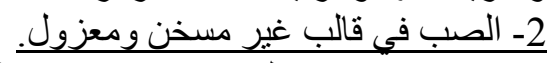

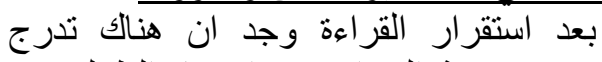

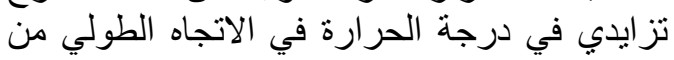

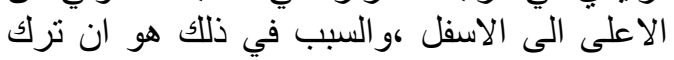

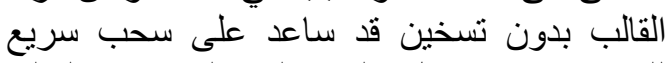

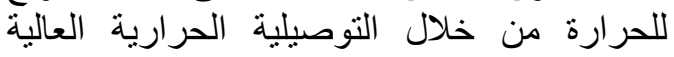

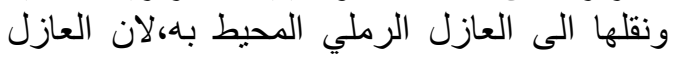

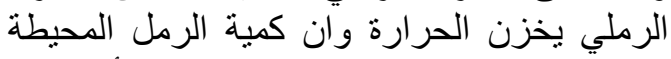

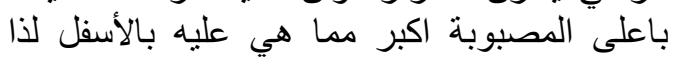

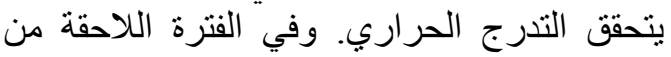

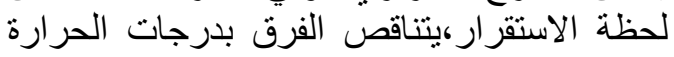

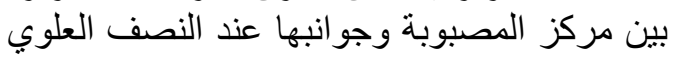

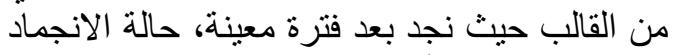

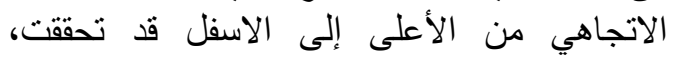

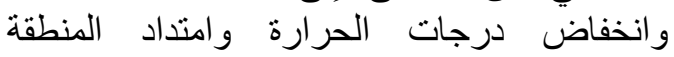

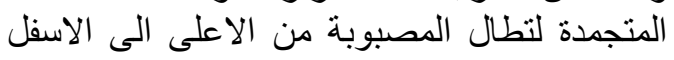

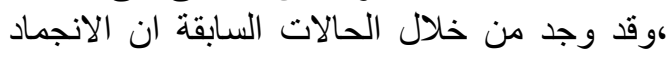

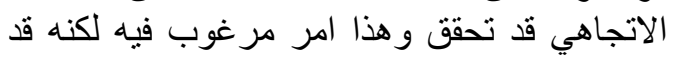

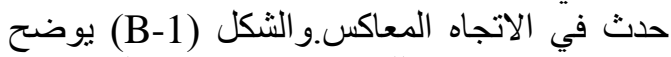

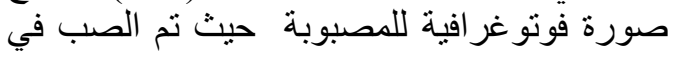




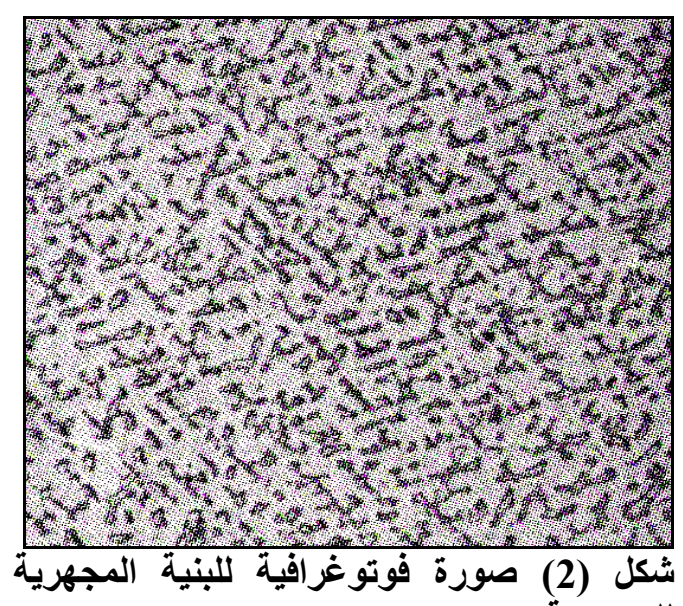

للمصبوية (A) (2) صورة

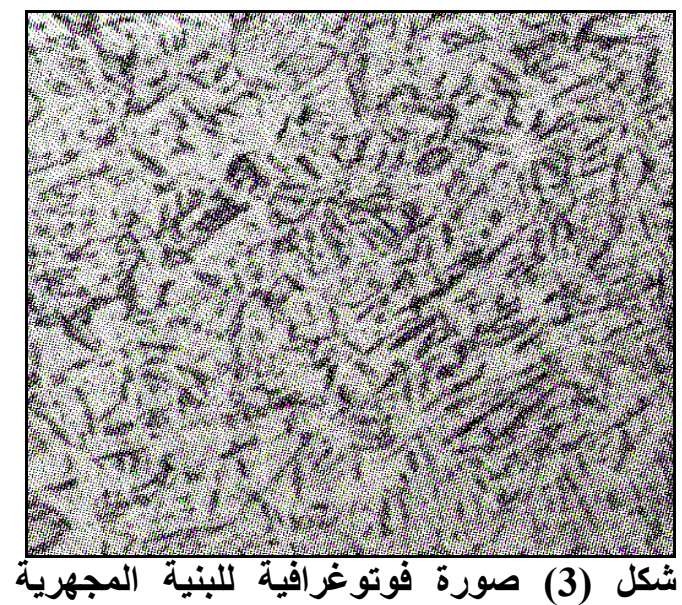

للمصبوبة (B) (B) صورن

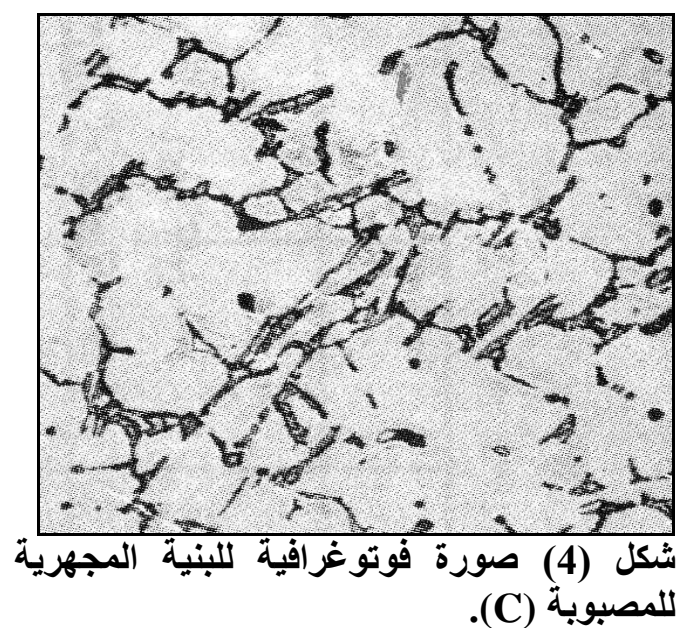

اما المسبوكة التي تم صبها في قالب غير مسخن

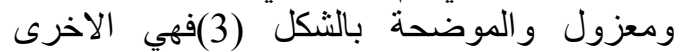
عبارة عن نركيب شجيري وحبيبات ذات نركيب

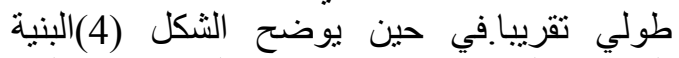
المجهرية لمسبوكة تم صبها في قالب مسخن ولكن ولكن

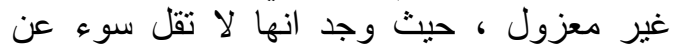

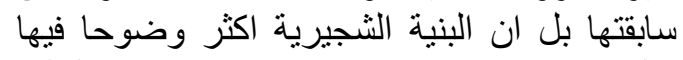

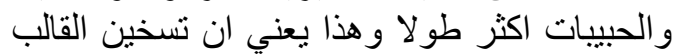

أكبر مما هو عليه بالاتجاهات الاخرى ،و هذه نتيجة

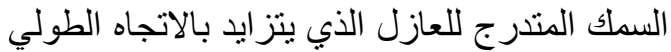

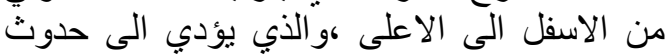

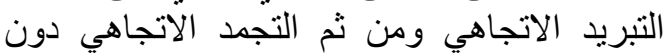

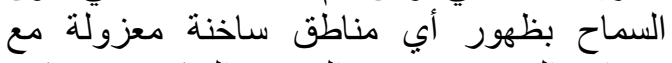
ضمان التعويض عن المعدن المنكمش بتغذيته

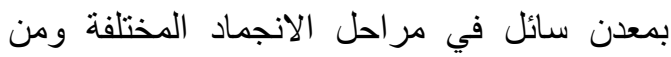
الجدير بالملاحظة ان فيل فل العازل العازل في توجيه الانجماد و السبطرة على اتجاهل قد تحقق بعد تسخين

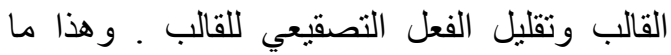
يوضحه الثكل (D-1) الذي يبين توزيع درجات الحرارة للمصبوبة التي تم صبها في قالب مسخن درجن

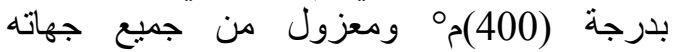
ولفترات زمنية مختلفة ،حيث يلاحظ ان الفرد الفرق في

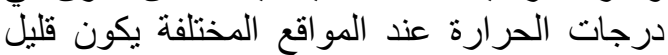

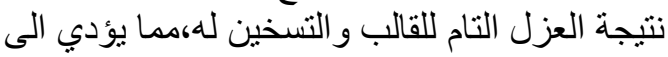
تقليل لفعل التصقيعي Chilling Effect التى اقل لتل

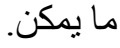

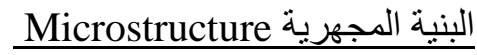
Dendrite يمكن القول ان البنية الثجيرية Structure

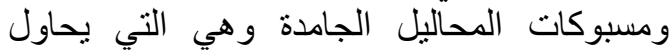

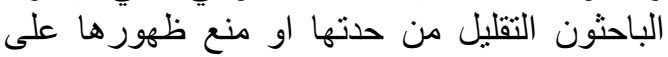

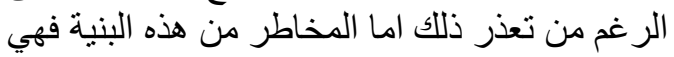

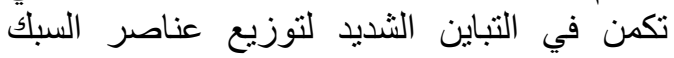

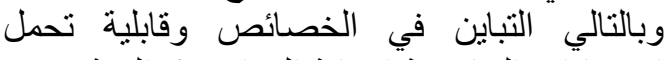
الاجهادات الخارجية اضافة الى ان هذه البنية البنية تصنع

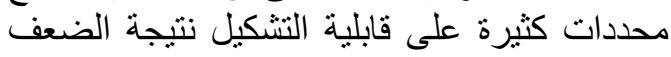

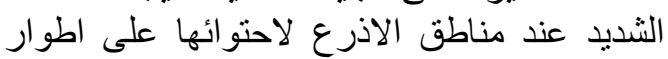

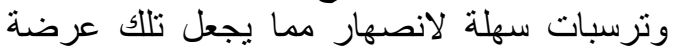
للتشقق على الساخن .لذا تجرى في بعض الان الاحيان

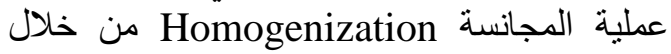
المعاملة الحرارية لغرض العادية العادة نوزيع عناصر

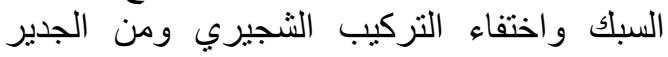
بالذكر ان تلاك العملية تحتاج الى وقت والت وتكاليف

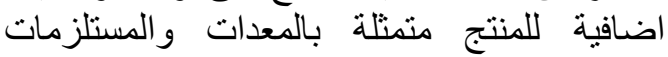

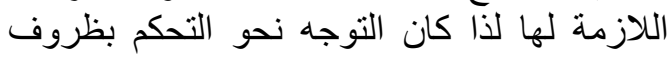

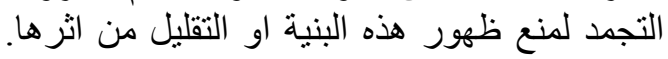

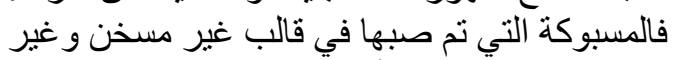
معزول لم تجرى لها أي معالجات على القيل القالب وقد

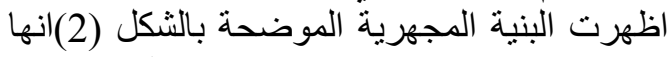

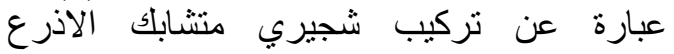

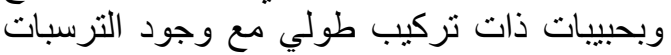
عن الحدود البلورية وهي من البنى المجهرية الغير لتردي

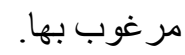


،وقد وجد بان الفجوة الثنانوية المتكونة في هذه

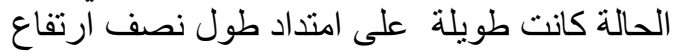

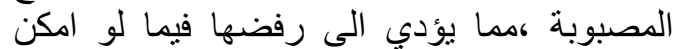

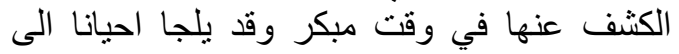
عمليات المجانسة ومن ثم التشكيل على ولى الساخن

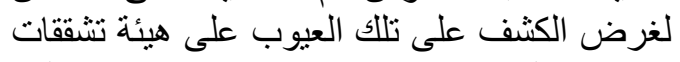

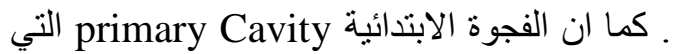

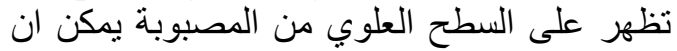

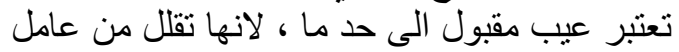
الانتفاع من طول المصبوبة .لذا فان التهان المصبوبة

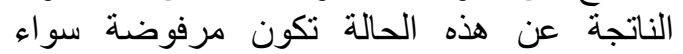

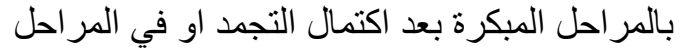

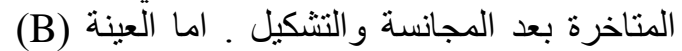

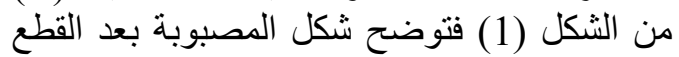

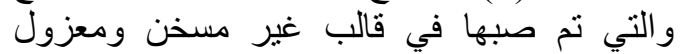

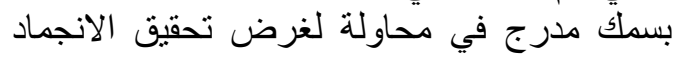
الاتجاهي وبالفعل تم تحقيق الانجماد الاتجاهي الاني

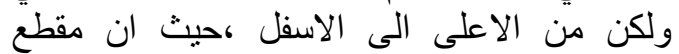

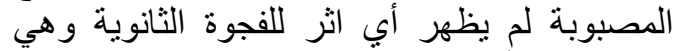
تعتبر حالة أفضل من السابق لكن ظهور الترة الفجوة الابتدائية بحجم وطول كبير وتحقيق الانجماد

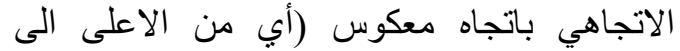

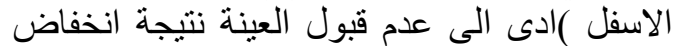

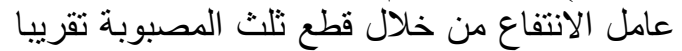

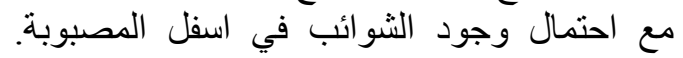

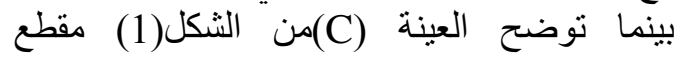

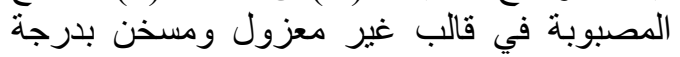
(400)مه لغرض التقليل من الفعل التصقيعي للقالب

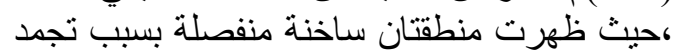
المعدن في وقت متاخر ،حيث كانت على هين هيئة

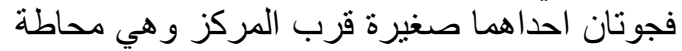

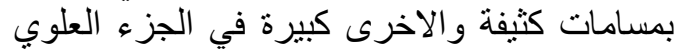

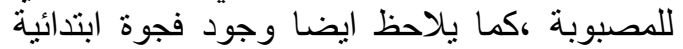

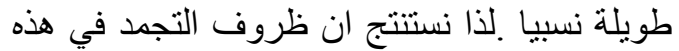

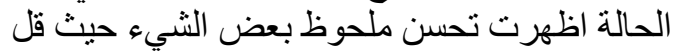

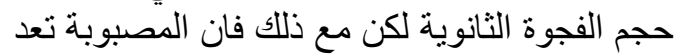

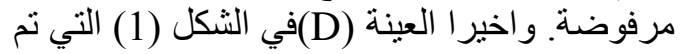

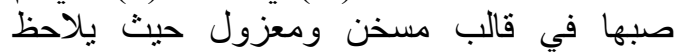
الانعدام ألتام للفجوة الثانوية والابتدائية وتحقيق عامل الانتفاع بنسبة (100\%) تقريبا ومن ثم تقليل

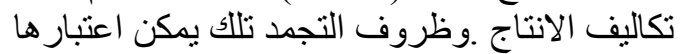

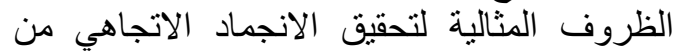

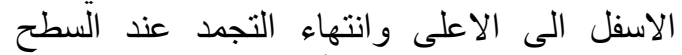

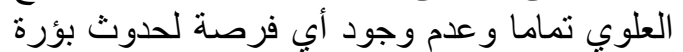

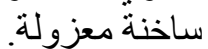

1. ان الصب في قالب فير فير مسخن و غير معزول

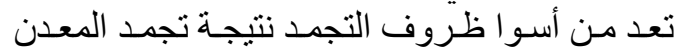

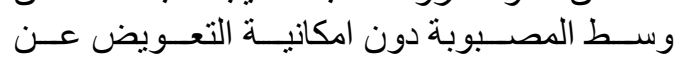

وحده لايجدي نفعا في تحسين البنية او الخصائص

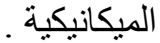
اما الثكل(5)فيوضح البنية المجهرية للمسبوكة الني

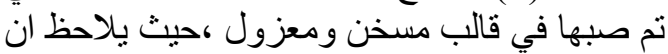
البنية هي عبارة عن حبيبات منساوية المحاور

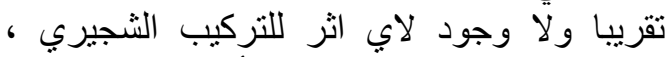

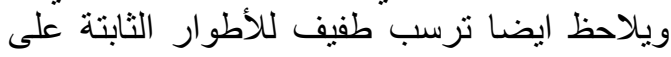

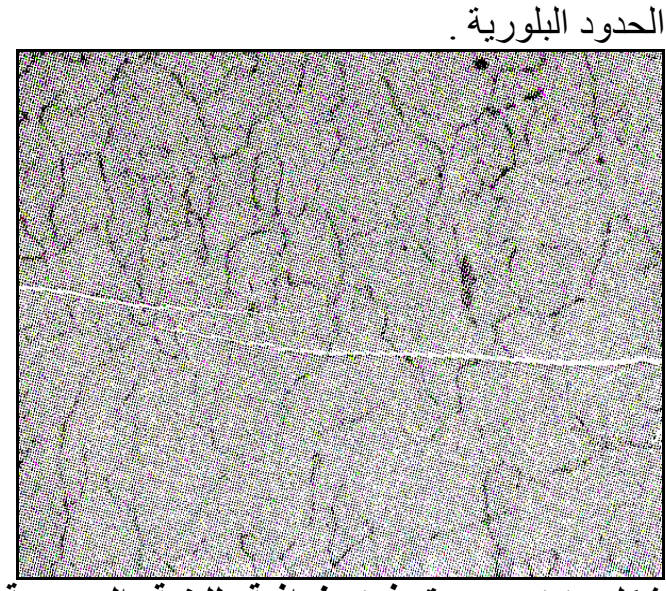

شكل (5) صورة فوتوغرافية للبنية المجهرية

للمصبوبة (D) (D)

من هنا نستنتج ان تسخين القالب بمفرده بدون عزل ليس له أي اثر في منع ظهور البنية الثجيرية

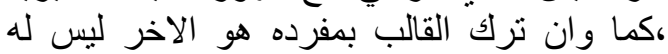

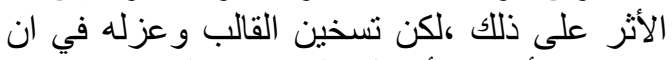

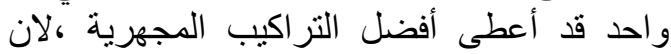

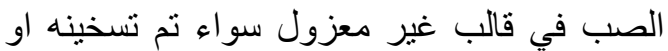

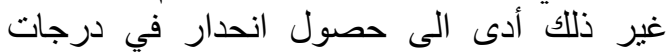

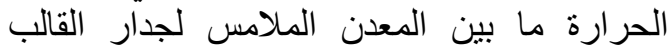

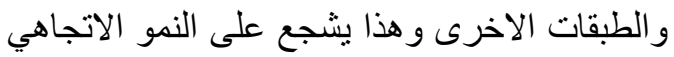

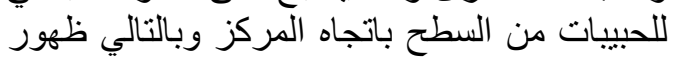

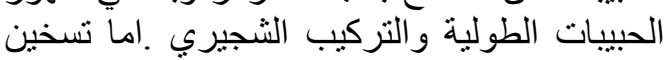

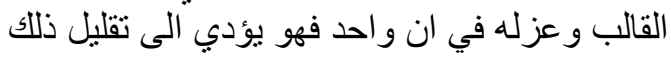

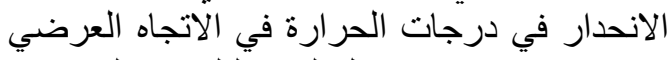

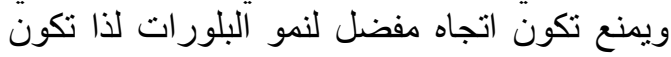

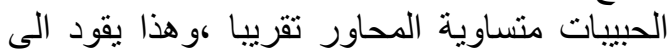

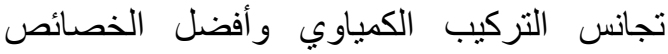

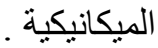

عيوب المصبوبات Defects of Ingots تم تقطيع الصبات بعني عند المستوى المركزي

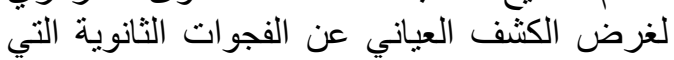

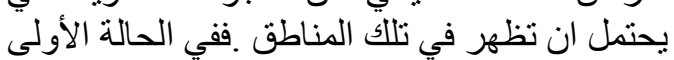

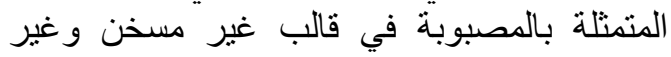

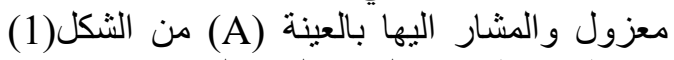

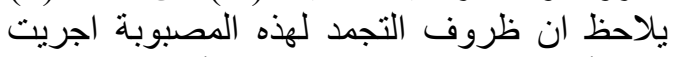
دون أي معالجة على القالى القالب مما أدى الى لى حتمية

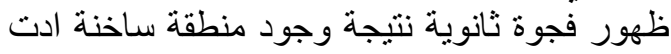

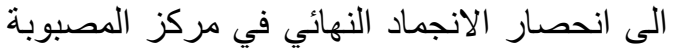


selected by Metals Hand book , volume 15 , Ninth Edition .

5- A.K.Singh , R.Pardeshi and B.Bash,

(Modeling of Convection During Solidification of Metal ), Sadhana ,2001, Vol.26. part 1\&2 FebruaryApril, P.139-162.

6- S.G.Humberto and D.F.Osmario, (Simulation of the Mushy Zone Solidification of a Binary Alloy),2000 , European Congress on Computational Methods in Applied Sciences and Engineering, Barcelona, September, P.1-12.

7- ASM international 1989 ," The Influence and control of porosity and Inclusions in Aluminum castings Aluminum Alloy Castings", properties processes and Applications . B. Cantor.

8- 2001, "Microstructure Development During Rapid Solidification". Kun Dar Li and Edward change American foundry

9- Society , 2009 " Explanation of The porosity Distribution in A206 Aluminum Alloy Castings" .J . P Anson, T . E

10- 2010, Gruzleski " The Eeffect of Hydrogen content on Relative Shrinkage and Gas Microporosity in Al- 7\% Si Casting". kun - Davli Ming cheng and Edward chang,

11- 2001 " Effect of pressure on the Feeding Behavior of A 356 Alloy in low Pressure Casting", American foundry society .

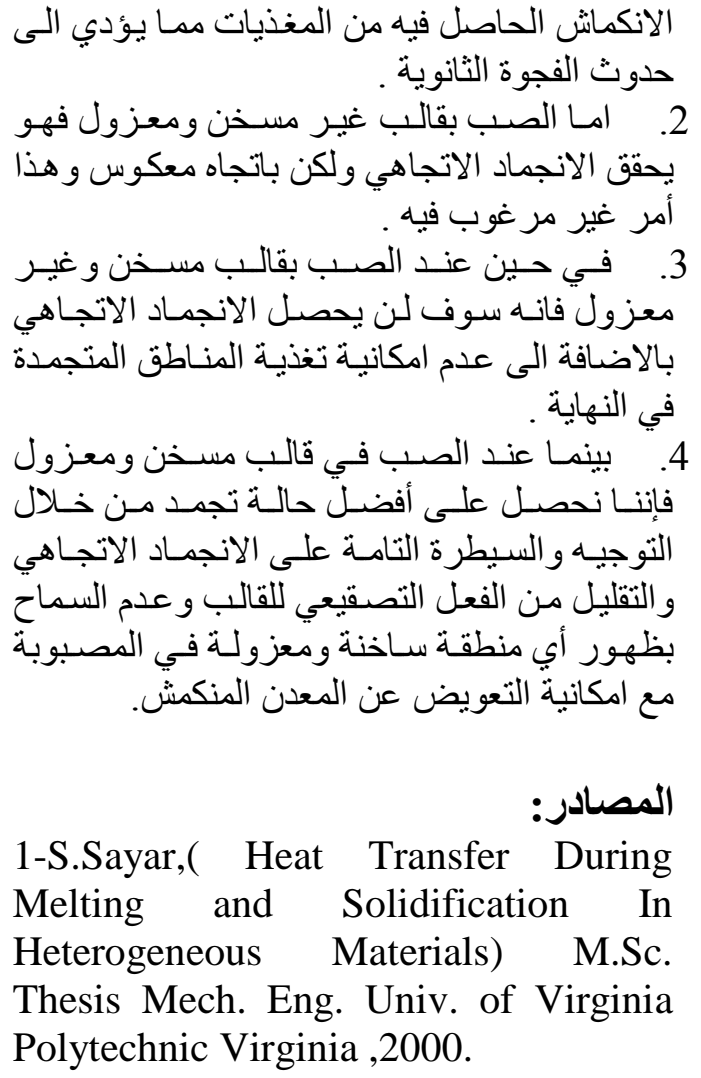

1-S.Sayar,( Heat Transfer During Melting and Solidification In Heterogeneous Materials) M.Sc. Thesis Mech. Eng. Univ. of Virginia Polytechnic Virginia ,2000.

2- S.T. Mclain , 2003 , American Foundry Society, "Study of Porosity and Solidification and its Effect on Porosity formation in Aluminum Alloy " Pore Morphology in Aluminum A 356 ", step Casting .

3-K.Y.Lee , S.M.Lee and C.P.Hong ,(Modeling of Fluid Flow and Solidification Grain Structures of AL$\mathrm{Cu}$ Crystalline Ribbons in Planar Flow Casting) 2001 .

4- J. lnst . Met , 2009 , " Secondary Hydrogen Porosity in Aluminum ", 


\title{
Ingots Defects under the influence of Solidification and Freezing Conditions.
}

\section{Farouk Mahdi Mansour* Mustafa A. Rijab Al-Najar*** \\ Amera kanan Asfur***}

*Assistant Professor/University of Tikrit - Faculty of Engineering. **Assistant Professor/ Technical Institute-Baquba /Mechanical Department

*** Assistant Lecturer / Faculty of Science for Girls

\begin{abstract}
:
The results of research to reach the conditions that prevents the emergence of primary or secondary voids and achieve worker benefit from molded by almost $100 \%$, which was the situation that cast poured in a mold heated and insulated from all sides to achieve freezing directional full starting from the region remote from the casting and ending then. Has also been compared to the microscopic structure of the resulting castings of various molding conditions, as these conditions have achieved the best sound microscopic structures.
\end{abstract}

